\title{
COMPORTAMENTO TÉRMICO DA CAULINITA HIDRATADA
}

\author{
José Eduardo Gardolinski, Harley Paiva Martins Filho e Fernando Wypych* \\ Departamento de Química, Universidade Federal do Paraná, CP 19081, 81531-990 Curitiba - PR
}

Recebido em 23/11/01; aceito em 28/5/02

\begin{abstract}
THERMAL BEHAVIOR OF HYDRATED KAOLINITE. Well-ordered kaolinite from the Brazilian Amazon Region (State of Pará) was initially reacted at $60{ }^{\circ} \mathrm{C}$ with a water dimethylsulfoxide mixture. After washing and characterisation, the resulting material was washed several times with methanol and in the final step with water. The water molecules displace the previously dimethylsulfoxide intercalated molecules and two different hydrated kaolinites were obtained. An unstable phase characterized by an interplanar basal distance of $0,996 \mathrm{~nm}$ that after drying collapse to the stable $0,844 \mathrm{~nm}$ hydrated kaolinite. The dehydration of the sample to disordered kaolinite was accompanied by Powder X-ray Diffractometry, thermal analysis (simultaneous TG and DSC) and FTIR spectroscopy.
\end{abstract}

Keywords: kaolinite; clay minerals; intercalation compounds.

\section{INTRODUÇÃO}

Os argilominerais do grupo da caulinita envolvem quatro variedades politípicas polimórficas, a Nacrita (JCPDS:16-606), Diquita (JCPDS:10-446), Caulinita (JCPDS:14-164 e 29-1488) e Haloisita $0,7 \mathrm{~nm}$ ou metahaloisita (JCPDS:29-1487), todos com a fórmula $\mathrm{Al}_{2} \mathrm{Si}_{2} \mathrm{O}_{5}(\mathrm{OH})_{4}$, além da Haloisita $1,0 \mathrm{~nm}$ ou endelita (JCPDS:1489) com a fórmula $\mathrm{Al}_{2} \mathrm{Si}_{2} \mathrm{O}_{5}(\mathrm{OH})_{4} \cdot 2 \mathrm{H}_{2} \mathrm{O}^{1,2}$. Tipicamente os argilominerais nacrita, diquita, caulinita e haloisita $0,7 \mathrm{~nm}$ ocorrem na forma de cristais tabulares e a haloisita 1,0 nm na forma de tubos ou cilindros, que ocorrem pelo enrolamento das lamelas da caulinita.

Dependendo da sua gênese, a caulinita pode se apresentar com elevada, média e alta ordem estrutural ao longo do eixo "b" da estrutura triclínica (ou monoclínica), sendo baixo o teor de substituintes isomórficos (normalmente $\mathrm{Fe}^{+3,+2}$ no sítio do alumínio), sendo considerada eletricamente neutra. Minerais do grupo da caulinita e outros argilominerais são formados pela decomposição de feldspatos por processos geológicos, tendo provavelmente sericita como intermediário (Eq. 1).

$2 \mathrm{KAlSi}_{3} \mathrm{O}_{8}+3 \mathrm{H}_{2} \mathrm{O} \rightarrow \mathrm{Al}_{2} \mathrm{Si}_{2} \mathrm{O}_{5}(\mathrm{OH})_{4}+4 \mathrm{SiO}_{2}+2 \mathrm{KOH}$

A formulação da caulinita pode ser descrita como resultante da deposição de 2 moles de gibbsita (variedade polimórfica do $\mathrm{Al}(\mathrm{OH})_{3}$ ) sobre dois moles de sílica, mantendo-se as estruturas dos dois compostos (tipo 1:1). A Figura 1 apresenta a estrutura da gibbsita (a), sílica (b), o processo de montagem da estrutura ideal da caulinita (c) e a estrutura final da caulinita (d).

Na estrutura da gibbsita os átomos de alumínio estão posicionados nos centros dos octaedros e, nos vértices, estão posicionados os grupamentos hidroxila. Somente $2 / 3$ dos octaedros são ocupados e os octaedros são ligados pelas arestas, o que caracteriza a caulinita como dioctaédrica ${ }^{1,2}$. Na estrutura da sílica, os átomos de silício estão posicionados nos centros dos tetraedros, nos quais os vértices são ocupados por átomos de oxigênio. Devido a forma com que os tetraedros são coordenados uns aos outros, cria-se um buraco na lamela que expõe a hidroxila interna para eventuais reações (Figura 1b).

*e-mail: wypych@quimica.ufpr.br

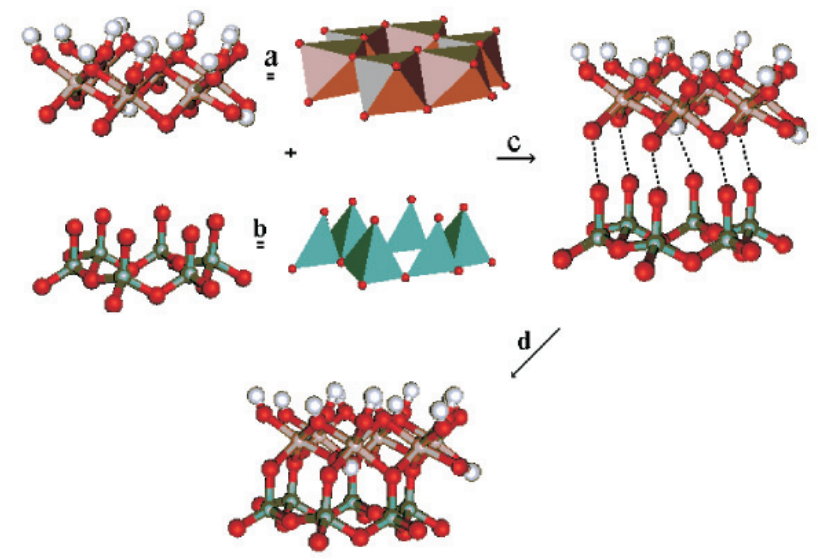

Figura 1. Estrutura da gibbsita (a), sílica (b), o processo de montagem da estrutura ideal da caulinita (c) e estrutura final da caulinita (d)

Exemplos de intercalação de caulinitas envolvem o uso de pequenas moléculas polares (tipicamente dimetilsulfóxido e $\mathrm{N}$ metilformamida $)^{3-9}$, fenômeno este associado ao processo de solvatação dos grupamentos hidroxila externos. A intercalação de algumas moléculas particulares somente é possível através do deslocamento de outras moléculas previamente intercaladas ${ }^{10-16}$. Recentemente, a intercalação de polímeros também foi reportada ${ }^{12,17-20}$ utilizando-se a metodologia de intercalação do precursor seguida de polimerização térmica ${ }^{12,20}$, intercalação do polímero fundido com caulinita previamente expandida ${ }^{17,18}$, ou deslocamento de moléculas de água previamente intercaladas ${ }^{19,20}$.

Um dos derivados obtidos durante o processo de lavagem da caulinita previamente intercalada é a caulinita hidratada ${ }^{21-23}$. Não existe um consenso na literatura sobre a composição química da caulinita hidratada $^{21-28}$ sendo sugerido, para alguns casos, o processo de funcionalização com grupamentos metóxido ${ }^{24}$, porém a caulinita hidratada foi preparada sem o uso do metanol e também tem características idênticas ${ }^{21}$.

Como a composição química da caulinita hidratada é semelhante à da haloisita ${ }^{29}$, o presente trabalho reporta o processo de preparação da caulinita hidratada e estudo do seu comportamento térmico. 


\section{PARTE EXPERIMENTAL}

A caulinita utilizada foi fornecida pelo Centro de Pesquisas da Petrobrás (CENPES - Rio de Janeiro) e é proveniente da bacia do Rio Capim, no Estado do Pará. A caulinita possui uma cor branca amarelada, uma elevada ordem estrutural e está contaminada com uma pequena concentração de titânio estrutural e ferro estrutural e não estrutural. O nível de impurezas situa-se em torno de 3\%, como determinado por experimentos de fluorescência de raios-X e ressonância paramagnética eletrônica ${ }^{30,31}$. Não existe nenhuma impureza cristalina que possa ser detectada, já que nos experimentos de difratometria de raios- $X$ somente reflexões correspondentes à caulinita foram observadas.

\section{Derivado intercalado com dimetilsulfóxido ${ }^{4,6}$}

Cerca de $9 \mathrm{~g}$ de caulinita (sem purificação prévia) foram suspensos em uma mistura de $60 \mathrm{~cm}^{3}$ de dimetilsulfóxido (DMSO - Merck PA) e $5,5 \mathrm{~cm}^{3}$ de água destilada. A suspensão resultante foi agitada a $60{ }^{\circ} \mathrm{C}$ por um período de 10 dias. O material resultante (K-DMSO) foi centrifugado a $4500 \mathrm{rpm}$ e seco a $50{ }^{\circ} \mathrm{C}$ por um período de $24 \mathrm{~h}$, para retirada do excesso de DMSO.

\section{Derivado intercalado com água e metanol ${ }^{11,13}$}

Imediatamente após o processo de secagem e caracterização do material intercalado com DMSO, 2,0 g deste reagiram com $50 \mathrm{~cm}^{3}$ de metanol (Merck PA) (sob agitação) por um período de $24 \mathrm{~h}$. O material resultante foi centrifugado a $4500 \mathrm{rpm}$ e o processo repetido. Após a última centrifugação, o material foi lavado oito vezes com metanol, o qual foi agitado duas vezes com $50 \mathrm{~cm}^{3}$ de água destilada, à temperatura ambiente, por $2 \mathrm{~h}$ e centrifugado. Em seguida o material foi caracterizado por difratometria de raios-X, ainda úmido (K-um) e seco por $24 \mathrm{~h}$ ao ar (K-ar), à temperatura ambiente. Para efeitos de comparação, o mesmo experimento foi realizado com caulinita pura, não sendo observado nenhum processo de intercalação.

\section{Instrumentos}

Para as medidas de difratometria de raios-X, filmes orientados do material foram obtidos sobre porta-amostras de vidro neutro. As medidas foram realizadas em um difratômetro Rigaku, pelo modo reflexão (radiação $\mathrm{Co}_{\mathrm{K} \alpha}=0,17902 \mathrm{~nm}, 40 \mathrm{kV}$ e $20 \mathrm{~mA}$, filtro de $\mathrm{Ni}$, velocidade de varredura de $1 \% \mathrm{~min}$ ). Para a retirada de radiações indesejáveis posicionou-se, entre a amostra e o detetor, um monocromador de grafite construído no próprio laboratório (LORXI - Departamento de Física da UFPR).

As medidas simultâneas de termogravimetria (TG) e calorimetria diferencial de varredura (DSC) foram realizadas em um equipamento Netzsch (modelo STA 409 Série EP) em atmosfera estática de ar, utilizando-se cadinhos de alumina de $0,065 \mathrm{~cm}^{3}$ e cadinhos de alumina vazios como referência. Cerca de $15 \mathrm{mg}$ de amostra foram analisados com uma velocidade de varredura de $5{ }^{\circ} \mathrm{C} / \mathrm{min}$ na faixa de 30 a $1000{ }^{\circ} \mathrm{C}$ utilizando-se uma curva de calibração obtida nas mesmas condições, com os cadinhos vazios, a qual foi subtraída das curvas experimentais.

Os espectros na região do infravermelho foram obtidos em um espectrofotômetro com rede de diodos BOMEM MICHELSON série MB100. Para isso, o material foi moído com $\mathrm{KBr}$ seco e analisado pelo modo transmissão, na faixa de 400 a $4000 \mathrm{~cm}^{-1}$, com resolução de $2 \mathrm{~cm}^{-1}$ e acumulação de 16 espectros.

Para o estudo do comportamento térmico da caulinita hidratada resultante da secagem ao ar (fase de $0,84 \mathrm{~nm}$ ), a mesma amostra foi aquecida em tempos acumulados em intervalos de $50{ }^{\circ} \mathrm{C}$ por $1 \mathrm{~h}$, entre 50 e $250{ }^{\circ} \mathrm{C}$. A amostra descrita como tratada à $250{ }^{\circ} \mathrm{C}$ foi aquecida durante $1 \mathrm{~h}$ à $50{ }^{\circ} \mathrm{C}, 1 \mathrm{~h}$ à $100{ }^{\circ} \mathrm{C}, 1 \mathrm{~h}$ à $150{ }^{\circ} \mathrm{C}$ e assim sucessivamente. Após cada tratamento térmico, parte da amostra foi retirada e submetida as análises de FTIR e difratometria de raios-X.

\section{RESULTADOS E DISCUSSÃO}

A Figura 2 apresenta os difratogramas de raios-X, obtidos na forma de filmes orientados sobre porta-amostras de vidro. As distâncias interplanares da caulinita, derivado intercalado com dimetilsulfóxido, caulinita hidratada úmida e seca são idênticas àqueles reportados anteriormente na literatura ${ }^{6,9,11,16,20,22}$ (Tabela 1). Na caulinita hidratada instável (K-um com distância interplanar basal de $0,996 \mathrm{~nm})$, a variação da distância interplanar de 0,279 nm (em relação à caulinita $=0,717 \mathrm{~nm}$ ) é compatível com o posicionamento da molécula de água entre as lamelas (diâmetro nominal da molécula de água $=0,28 \mathrm{~nm}^{32}$ ). Essa condição é metatestável colapsando a fase hidratada estável (K-ar), com uma distância interplanar de 0,844 $\mathrm{nm}$. Como nesse caso a expansão interplanar de $0,127 \mathrm{~nm}$ é menor que o diâmetro das moléculas de água, é bastante provável que essas moléculas de água estejam alocadas no buraco hexagonal criado pela

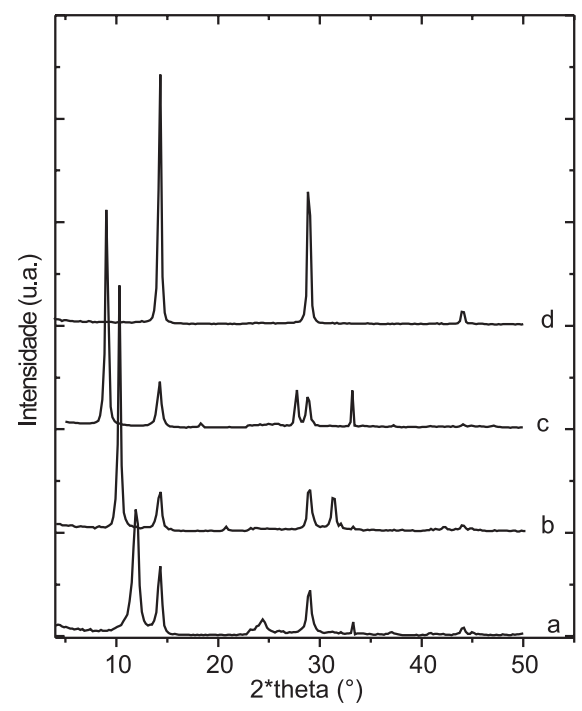

Figura 2. Difratogramas de raios-X da fase hidratada seca ao ar por $24 \mathrm{~h}$ (a), fase hidratada ainda úmida (b), caulinita intercalada com DMSO (c) $e$ caulinita pura (d). Silício como padrão interno indicado por *

Tabela 1. Identificação das fases (Índice de reação - IR), espaçamentos interplanares basais $d_{001}(\mathrm{~nm})$ e variações dos espaçamentos interplanares $\Delta \mathrm{d}(\mathrm{nm})$ em relação à caulinita pura

\begin{tabular}{lcc}
\hline Fase $(\operatorname{IR} \%)$ & $\mathrm{d}_{001}(\mathrm{~nm})$ & $\Delta \mathrm{d}(\mathrm{nm})$ \\
\hline $\mathrm{K}$ & 0,717 & 0 \\
D-DMSO (83,5) & 1,121 & 0,404 \\
K-um. $(84,8)$ & 0,996 & 0,279 \\
K-ar $(58,3)$ & 0,844 & 0,127 \\
$50^{\circ} \mathrm{C}$ & 0,83 & 0,113 \\
$100^{\circ} \mathrm{C}$ & 0,80 & 0,0083 \\
$150^{\circ} \mathrm{C}$ & 0,77 & 0,053 \\
$200^{\circ} \mathrm{C}$ & 0,74 & 0,23 \\
$250^{\circ} \mathrm{C}$ & 0,717 & 0 \\
\hline
\end{tabular}


ligação dos tetraedros de silício ${ }^{25}$ da lamela da caulinita (Figura 1b). Após tratamento térmico a ordem estrutural é diminuída como observado pela presença de uma banda na forma de um "dente de serra" na região de 22 a $27^{\circ}$ em $2 * \theta^{1,33}$ (Figura 4e e 4f).

A Figura 3 apresenta medidas de TG/DSC da caulinita pura (a) e $\mathrm{K}$-ar (b). A amostra de K-um não pode ser medida porque somente existe se for mantida molhada. Os valores das temperaturas foram determinados nos máximos e mínimos dos picos das curvas de DSC.

Observa-se que a caulinita pura (Figura 3a) apresenta um pico endotérmico centrado em $51{ }^{\circ} \mathrm{C}$, correspondente à eliminação de moléculas de água de adsorção/absorção e um pico endotérmico centrado em $529^{\circ} \mathrm{C}$, correspondente a seu processo de desidroxilação, o qual transforma a caulinita em metacaulinita ${ }^{1,34,35}$. Um pequeno pico endotérmico foi observado em $275^{\circ} \mathrm{C}$, provavelmente de uma impureza cristalina, apesar de que por difratometria de raios- $\mathrm{X}$, nenhum material cristalino contaminante foi observado. $\mathrm{O}$ pico exotérmico em $977{ }^{\circ} \mathrm{C}$ é relacionado com a nucleação da mulita ${ }^{33}$. Descontando-se a massa de água adsorvida (pico endotérmico em $51{ }^{\circ} \mathrm{C}$ ), a perda de massa relativa à desidroxilação de $13,6 \%$ está em boa concordância com o valor esperado de 13,96\% (considerandose que o resíduo consiste de $\mathrm{Al}_{2} \mathrm{O}_{3}$ e $2 \mathrm{SiO}_{2}$ ). Constata-se desta forma que a caulinita original é bastante pura com teor de impurezas menores que $3 \%$, como confirmado por difratometria de raios-X, ressonância paramagnética eletrônica e fluorescência de raios- $\mathrm{X}^{30,31}$.

a)

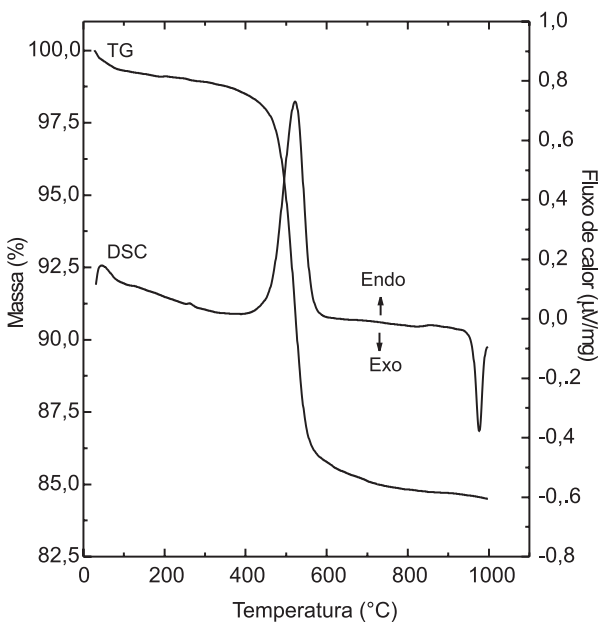

b)

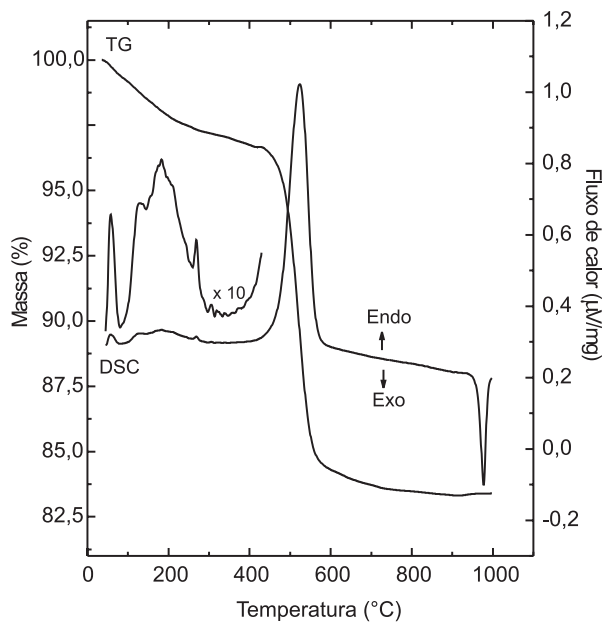

Figura 3. Curvas de TG/DSC da caulinita (a) e caulinita hidratada seca ao ar por $24 h(b)$
Desconsiderando-se que a amostra tenha moléculas de água de adsorção/absorção, a caulinita hidratada seca ao ar (K-ar)(Figura 3b) apresenta uma perda de massa contínua até cerca de $300^{\circ} \mathrm{C}$, associada a um pico endotérmico em $60^{\circ} \mathrm{C}$ e um pico largo entre 100 e $300{ }^{\circ} \mathrm{C}$. A seguir observou-se um pico endotérmico em $518{ }^{\circ} \mathrm{C}$, atribuído ao processo de desidroxilação da matriz, o qual transforma a caulinita em metacaulinita ${ }^{1,34,35}$. O pico exotérmico em $977^{\circ} \mathrm{C}$ é idêntico àquele reportado para a caulinita (Figura 3a), relacionado com a nucleação da mulita ${ }^{33}$. Após a eliminação da água adsorvida/absorvida e água interlamelar à $300^{\circ} \mathrm{C}$, a caulinita hidratada transforma-se em caulinita, sendo os eventos térmicos idênticos à caulinita da Figura $3 \mathrm{a}$.

Considerando-se que a concentração de caulinita era de ordem de $41,7 \%$ e ausência de águas de adsorção/absorção, a perda de massa no primeiro processo até $350{ }^{\circ} \mathrm{C}$ de $3,07 \%$ corresponde à estequiometria de 0,80 moles de moléculas de água em relação à caulinita $\left(\mathrm{Al}_{2} \mathrm{SiO}_{5}(\mathrm{OH})_{4}\left(\mathrm{H}_{2} \mathrm{O}\right)_{0,80 \pm 0.04}\right)$. Esse valor é igual ${ }^{23}$ ou significativamente inferior aos valores reportados anteriormente ${ }^{27,28}$. O resíduo total de $83,1 \%$ está em boa concordância com o resíduo experimental de $83,5 \%$ para a fórmula proposta. Considerando-se a perda de moléculas de água adsorvidas/absorvidas até $100{ }^{\circ} \mathrm{C}$, a estequiometria determinada será $\mathrm{Al}_{2} \mathrm{SiO}_{5}(\mathrm{OH})_{4}\left(\mathrm{H}_{2} \mathrm{O}\right)_{0,55+0,03}$ e o resíduo experimental será de $83,9 \%$ e o esperado de $84,2 \%$, ambos em relação a amostra seca. $\mathrm{O}$ valor calculado considerando-se a presença de água adsorvida/absorvida aproxima-se mais dos valores reportados mais recentemente $e^{21,22}$.

Para o caso da intercalação de N-metilformamida e lavagem do material intercalado com metanol para a obtenção de caulinita hidratada, foi proposto o processo de funcionalização parcial dos grupamentos hidroxila com grupamentos metóxido ${ }^{24}$. Embora no presente caso o metanol tenha sido utilizado no processo de lavagem, a mesma fase hidratada pode ser obtida através da lavagem com água da fase intercalada com uréia ${ }^{21}$ descartando-se, desta forma, a funcionalização com grupamentos metóxido. Outra evidência da não existência do grupamento metóxido é a não observação de bandas características através de FTIR $^{36}$.

A Figura 4 apresenta os difratogramas de raios- $X$ da caulinita hidratada seca ao ar (K-ar) e após aquecimento a varias temperaturas (Tabela 1).

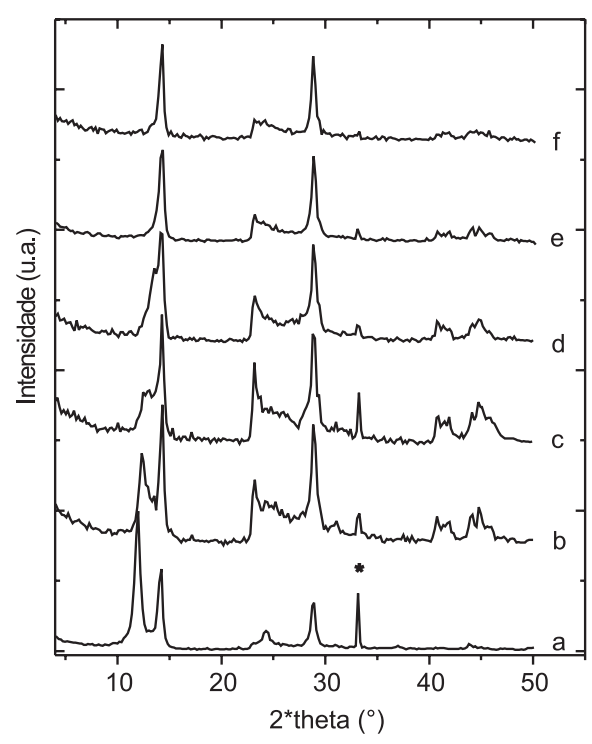

Figura 4. Difratogramas de raios- $X$ da caulinita hidratada (a) e após aquecimento a $50{ }^{\circ} \mathrm{C}(\mathrm{b}), 100{ }^{\circ} \mathrm{C}(\mathrm{c}), 150{ }^{\circ} \mathrm{C}(\mathrm{d}), 200{ }^{\circ} \mathrm{C}(e)$ e $250{ }^{\circ} \mathrm{C}(\mathrm{f})$. Silício como padrão interno indicado por * 
Observa-se que, com o aumento da temperatura o teor de caulinita hidratada e a distância interplanar basal dos produtos intermediários são gradativamente reduzidos, até a obtenção da caulinita com desordem estrutural, à temperatura de $250^{\circ} \mathrm{C}$.

O passo abrupto de 0,152 nm (Figura 5) perdido após secagem ao ar da amostra K-um é menor que o diâmetro da molécula de água (diâmetro de Van der Waals da molécula de água = 0,28 nm). Essa é uma evidência de que na fase de 0,996 nm (K-um - Figura 6a), duas moléculas de água não equivalentes estão interagindo e posicionadas nos espaçamentos interlamelares. No momento da retirada da primeira molécula, a segunda permanece parcialmente no interior do buraco (Figura 6b). Como na fase de $0,844 \mathrm{~nm}$, a variação da distância interplanar é de $0,127 \mathrm{~nm}$ e o diâmetro da molécula de água é de $0,28 \mathrm{~nm}$, a profundidade do buraco pode ser calculada como sendo de $0,153 \mathrm{~nm}$. Os passos subsequentes ocorrem limitados pela velocidade de difusão das moléculas de água nos espaçamentos interplanares formando compostos intermediários.

No espectro da caulinita pura (Figura $7 \mathrm{~g}$ ), as bandas observadas em 3695,3667 e $3650 \mathrm{~cm}^{-1}$ são relativas às hidroxilas superficiais e

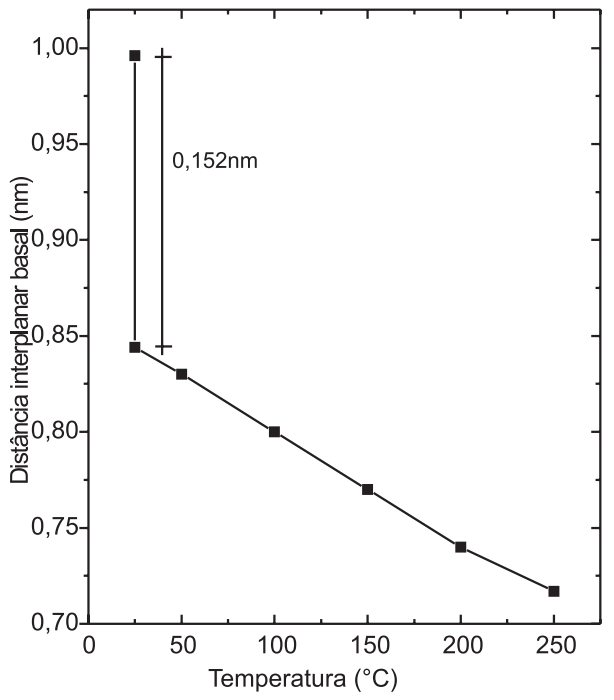

Figura 5. Variação da distância interplanar basal da caulinita hidratada com a temperatura

a

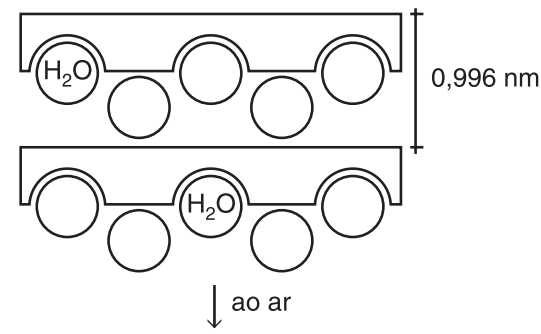

b

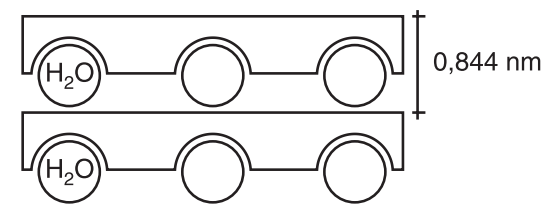

Figura 6. Representação esquemática do posicionamento das moléculas de água nos espaçamentos interlamelares da caulinita. Fase de 0,996 nm (a) $e$ $0,844 \mathrm{~nm}(b)$



Figura 7. FTIR da caulinita hidratada seca ao ar (a) e tratada a $50{ }^{\circ} \mathrm{C}(\mathrm{b})$, $100{ }^{\circ} \mathrm{C}(\mathrm{c}), 150{ }^{\circ} \mathrm{C}(\mathrm{d}), 200{ }^{\circ} \mathrm{C}(\mathrm{e})$ e $250{ }^{\circ} \mathrm{C}(\mathrm{f})$, além da caulinita pura $(\mathrm{g})$

a banda em $3619 \mathrm{~cm}^{-1}$, à hidroxila interna (Figura 1d) ${ }^{37-39}$. O espectro da caulinita hidratada apresenta bandas em 3695, 3667, 3650, 3619, 3598 e $3550 \mathrm{~cm}^{-1}$. Resultados semelhantes foram obtidos quando a amostra intercalada com $\mathrm{NMF}^{13}$ foi lavada com metanol, porém nesse caso a banda centrada em $3598 \mathrm{~cm}^{-1}$ não foi observada.

Quando a amostra intercalada com DMSO foi lavada várias vezes com metanol e água ${ }^{16}$, o espectro é semelhante porém com inversões nas intensidades das bandas. Quando a caulinita intercalada com uréia (ou etileno glicol) foi lavada com água ${ }^{21,22}$, os espectros de FTIR são idênticos. No início do processo de lavagem da amostra intercalada com etileno glicol, somente a banda em $3550 \mathrm{~cm}^{-1}$ foi observada, surgindo em seguida a banda em $3599 \mathrm{~cm}^{-1}$. Esse fenômeno foi associado à heterogeneidade da amostra ${ }^{22}$.

As bandas em 3550 e $3598 \mathrm{~cm}^{-1}$ podem ser interpretadas como sendo relacionadas com o posicionamento das moléculas de água interlamelares, interagindo com as hidroxilas internas e externas, através de ligações de hidrogênio. Após aquecimento a $50^{\circ} \mathrm{C}$ por $1 \mathrm{~h}$ (Figuras $7 \mathrm{~b}$ e $8 \mathrm{~b}$ ), o espectro de FTIR é muito semelhante ao anterior (Figura 7a) porém as bandas em 3667 e $3650 \mathrm{~cm}^{-1}$ na caulinita hidratada seca ao ar são desdobradas em duas bandas cada, aparecendo em 3666 e $3672 \mathrm{~cm}^{-1}$, e 3646 e $3653 \mathrm{~cm}^{-1}$, respectivamente. Essas novas bandas desaparecem simultaneamente ao desaparecimento das bandas em 3550 e $3598 \mathrm{~cm}^{-1}$. Essas bandas nunca foram descritas na literatura e são uma evidência a mais de que várias hidroxilas externas e inclusive a interna, estão envolvidas nas ligações de hidrogênio com a molécula de água intercalada. Essa novas bandas são, inclusive, observadas quando o espaçamento interplanar já foi reduzido conforme pode ser observado nas Figuras 4 e 5.

$\mathrm{O}$ efeito da redução da distância interplanar basal é muito afetado a $100{ }^{\circ} \mathrm{C}$ (Figura 7c), embora o espectro de FTIR ainda seja semelhante àquele da temperatura ambiente. Uma observação mais cuidadosa entretanto demonstra que as bandas situadas em 3598 e $3550 \mathrm{~cm}^{-1}$ tiveram suas intensidades diminuídas, tendo sido totalmente eliminadas a $200{ }^{\circ} \mathrm{C}$ (Figura 7e). Os espectros da amostra seca a 200 ou $250{ }^{\circ} \mathrm{C}$ são semelhantes aos da amostra da caulinita com exceção da banda em $3667 \mathrm{~cm}^{-1}$, que era observada na caulinita pura (Figura $7 \mathrm{~g}$ ) e não é mais observada (Figura 7e). Esse espectro é característico da caulinita com desordem estrutural ${ }^{39}$ demonstrando que o processo de intercalação do DMSO, substituição do DMSO 
pela água e remoção desta, provoca uma desordem estrutural na caulinita. No momento da eliminação da molécula de água, as duas bandas em 3550 e $3598 \mathrm{~cm}^{-1}$ são eliminadas do espectro de FTIR (Figura 7d-f) e o desdobramento das bandas é eliminado (Figura 8). O pequeno ombro situado em $3598 \mathrm{~cm}^{-1}$ observado na caulinita pura se deve à ligação $\mathrm{Fe}-\mathrm{O}-\mathrm{H}$ gerada após substituições isomórficas de átomos de alumínio octaédricos por átomos de ferro ${ }^{39}$.



Figura 8. Espectros de FTIR expandidos da caulinita hidratada seca ao ar (a), tratada a $50{ }^{\circ} \mathrm{C}\left(\right.$ b) e $100{ }^{\circ} \mathrm{C}(\mathrm{c})$, além da caulinita pura $(d)$

A região relativa às vibrações do esqueleto da caulinita é pouco afetada pelo processo de intercalação da molécula de água salvo algumas exceções. As bandas situadas em $913 \mathrm{~cm}^{-1}$, atribuída à hidroxila externa e $936 \mathrm{~cm}^{-1}$, atribuída à hidroxila interna sofrem pequenas alterações de intensidade e observa-se uma nova banda em $966 \mathrm{~cm}^{-1}$ na caulinita hidratada. Na caulinita de alta ordem estrutural a banda em $757 \mathrm{~cm}^{-1}$ é de maior intensidade que a banda em $792 \mathrm{~cm}^{-1}$ (atribuídas às deformações angulares das ligações Si-O-Si e Al-O-Si), essas bandas tornam-se praticamente de igual intensidade após aquecimento a $200{ }^{\circ} \mathrm{C}$, característica da caulinita com baixa ordem estrutural $^{39}$. Essa é uma evidência a mais de que não somente as hidroxilas internas e externas participam das ligações de hidrogênio mas também o anel hexagonal do silicato ${ }^{40}$.

\section{CONCLUSÕES}

Não existe a possibilidade de se proceder à intercalação de água diretamente na caulinita pura já que esta apresenta uma estrutura bastante coesa, estabilizada através das ligações de hidrogênio entre os grupamentos hidroxila ligados ao alumínio de uma lamela e átomos de oxigênio ligados ao silício de outra lamela. Através da metodologia conhecida como "método do deslocamento", intercalase previamente o DMSO, sendo que este pode ser posteriormente substituído por outras moléculas orgânicas ou inorgânicas.

Na caulinita hidratada instável, as moléculas de água são provavelmente posicionadas entre as lamelas, sendo retiradas após secagem ao ar, já que para todos os casos, o espaço ocupado pelas moléculas de água é menor que o valor esperado. É provável que as moléculas intercaladas estejam ligadas por ligações de hidrogênio às hidroxilas internas posicionadas no interior da cavidade gerada pelas ligações dos tetraedros da sílica (Figuras 1 e 6), além de hidroxilas específicas externas (fenômeno conhecido como "chaveamento").
Durante o processo de substituição das moléculas de DMSO, parte do material é regenerado na forma de caulinita, já que o índice de intercalação cai de $83,5 \%$ (com DMSO) e 58,3\% para o caso da intercalação de água. Estranhamente para o caso da intercalação do metanol, o índice de intercalação cresceu para 84,8\%, embora a variação esteja dentro do erro experimental do método.

Após o processo de intercalação e retirada das moléculas de água intercaladas com tratamento térmico, a caulinita sofre um processo de desordem estrutural, como observado por difratometria de raios$\mathrm{X}$ e FTIR.

Embora aparentemente, dentro do nosso conhecimento, nunca tenha sido reportado na literatura o processo natural de intercalação da caulinita, é bastante provável que algumas caulinitas naturais (talvez classificadas erroneamente por não possuírem o espaçamento interplanar basal característico de $0,72 \mathrm{~nm}$ ) consistam de caulinitas intercaladas e até funcionalizadas. Os compostos utilizados no processo de intercalação ou funcionalização poderiam ser derivados da decomposição de matéria orgânica natural como por exemplo, os ácidos húmicos ou ainda aqueles introduzidos pelo homem no cultivo de lavouras. Estudos estão em curso no sentido de identificar e caracterizar argilominerais intercalados (principalmente com água) e organo-funcionalizados, na natureza.

\section{AGRADECIMENTOS}

Ao Departamento de Física da UFPR pelo uso do difratômetro de raios-X, ao CNPq, à FINEP e Fundação Araucária.

\section{REFERÊNCIAS}

1. Santos, P. S.; Ciência e Tecnologia de Argilas, Edgard Blücher Ltda.: São Paulo, 1989, vol. 1.

2. Grim, R. E.; Clay Mineralogy, McGraw-Hill Book Company Inc.: New York, 1953.

3. Maxwell, C. B.; Malla, P. B.; Amer. Ceram. Soc. Bull. 1999, 57.

4. Thompson, J. G.; Cuff. C.; Clays Clay Miner. 1985, 33, 490.

5. Uwins, P. J. R.; Mackinnon, I. D. R.; Thompson, J. G.; Yago, A. J. E.; Clays Clay Miner. 1993, 41, 707.

6. Wypych, F.; Gardolinski, J. E.; Peralta-Zamora, P.; J. Colloid Interface Sci. 1999, 211, 137.

7. Frost, R. L.; Kristof, J.; Paroz, G. N.; Kloprogge, J. Y.; Phys. Chem. Miner. 1999, 26, 257

8. Olejnik, S.; Posner, A. M.; Quirk, J. P.; Clay Miner. 1970, 8, 421.

9. Tsunematsu, K.; Tateyama, H.; J. Am. Ceram. Soc. 1999, 82, 1589.

10. Gardolinski, J. E.; Ramos, L. P.; Pinto de Souza, G.; Wypych, F.; J. Colloid Interface Sci. 2000, 221, 284.

11. Komori, Y.; Sugahara, Y.; Kuroda, K.; J. Mater. Res. 1998, 13, 930.

12. Sugahara, Y.; Satokawa, S.; Yoshioka, K.; Kuroda, K.; Kato, C.; Clays Clay Miner. 1990, 2, 137.

13. Komori, Y.; Sugahara, Y.; Kuroda, K.; Appl. Clay Sci. 1999, 15, 241.

14. Sugahara, Y.; Kitano, S.; Satokawa, S.; Kuroda, K.; Kato, C.; Bull. Chem. Soc. Jpn. 1986, 59, 2607.

15. Tunney, J. J.; Detellier, C.; Can. J. Chem. 1997, 75, 1766.

16. Sato, M.; Clays Clay Miner. 1999, 6, 793.

17. Tunney, J. J.; Detellier, C.; Chem. Mater. 1996, 8, 927.

18. Wypych, F.; Gardolinski, J. E.; Carrera, L. C. M.; Cantão, M. P.; J. Mater. Sci. 1999, 35, 1 .

19. Komori, Y.; Sugahara, Y.; Kuroda, K.; Chem. Mater. 1999, 11, 3.

20. Komori, Y.; Sugahara, Y.; Kuroda, K.; J. Mater. Chem. 1999, 9, 3081.

21. Gardolinski, J. E.; Wypych, F.; Cantão, M. P.; Quim. Nova 2001, $24,761$.

22. Tunney, J.; Detellier, C.; Clays Clay Miner. 1994, 42, 473.

23. Wada, K.; Am. Miner. 1965, 50, 924

24. Komori, Y.; Enoto, H.; Takenawa, R.; Hayashi, S.; Sugahara, Y.; Kuroda, $\mathrm{K}$; Langmuir 2000, 16, 5506.

25. Jemai, S.; Amara, A. B. H.; Plançon, A.; J. Appl. Crystallogr. 2000, 33, 1075.

26. Raythatha, R.; Lipsicas, M.; Clays Clay Miner. 1985, 33, 333.

27. Contanzo, P. M.; Clemency, C. V.; Giese. Jr., R. F.; Clays Clay Miner. 1980, 28,155 . 
28. Wada, N.; Raythatha, R.; Minomura, S.; Solid State Commun. 1987, 63, 783.

29. Tari, G.; Bobos, I.; Gomes, C. S. F.; Ferreira, J. M. F.; J. Colloid Interface Sci. 1999, 210, 360.

30. Lombardi, K. C.; Guimarães, J. L.; Mangrich, A. S.; Mattoso, N.; Abbate, M.; Schreiner, W. H.; Wypych, F.; J. Braz. Chem. Soc., 2002, 13, 270.

31. Guimarães, J. L.; Peralta-Zamora, P.; Wypych, F.; J. Colloid Interface Sci. 1998, 206, 281.

32. Schöllhorn, R. Em Progress in Intercalation Research; Müller-Warmuth W.; Schöllhorn, R., eds.; Kluver Academic Publishers: Dordrecht, 1994

33. Gomes, C. F.; Argilas. O que são e para que servem, Fundação Calouste Gulbenkian: Lisboa, 1986.
34. Frost, R. L.; Vassallo, A. M.; Clays Clay Miner. 1996, 44, 635.

35. Okada, K.; Shimai, A.; Takei, T.; Hayashi, S.; Yasumori, A.; MacKenzie, J. D.; Microporous Mesoporous Mater. 1998, 21, 289.

36. Tunney, J. J.; Detellier, C.; J. Mater. Chem. 1996, 6, 1679.

37. Frost, R. L.; Clays Clay Miner. 1998, 46, 280.

38. Shoval, S.; Yariv, S.; Michaelian, K. H.; Lapides, I.; Boudeuille, M.; Panczers, G.; J. Colloid Interface Sci. 1999, 212, 523.

39. Russel J. D.; Fraser, A.R. Em Clay Mineralogy: Spectroscopic and Chemical Determinative Methods; Wilson, M.J., ed.; Chapman and Hall: London, 1994

40. Smirnov, K. S.; Bougeard, D.; J. Phys. Chem. 1999, 103, 5266. 